This is the peer-reviewed version of:

Cupini, Giovanni, e Ermanno Lanconelli. «Densities with the Mean Value Property for Sub-Laplacians: An Inverse Problem». In Harmonic Analysis, Partial Differential Equations and Applications, a cura di Sagun Chanillo, Bruno Franchi, Guozhen Lu, Carlos Perez, e Eric T. Sawyer, 109-24. Applied and Numerical Harmonic Analysis. Cham: Springer International Publishing, 2017.

The final published version is available online at: https://doi.org/10.1007/978-3319-52742-0_8

Rights / License:

The terms and conditions for the reuse of this version of the manuscript are specified in the publishing policy. For all terms of use and more information see the publisher's website.

This item was downloaded from IRIS Università di Bologna (https://cris.unibo.it/)

When citing, please refer to the published version. 


\title{
DENSITIES WITH THE MEAN VALUE PROPERTY FOR SUB-LAPLACIANS: AN INVERSE PROBLEM
}

\author{
Dedicated to Richard L. Wheeden \\ Giovanni Cupini - Ermanno Lanconelli \\ Dipartimento di Matematica, Università di Bologna \\ Piazza di Porta S. Donato 5, 40126 Bologna, Italy \\ e-mail: giovanni.cupini@unibo.it, ermanno.lanconelli@unibo.it
}

\begin{abstract}
Inverse problem results, related to densities with the mean value property for the harmonic functions, were recently proved by the authors. In the present paper we improve and extend them to the sub-Laplacians on stratified Lie groups.
\end{abstract}

\section{INTRODUCTION}

Let $\Omega$ be an open subset of $\mathbb{R}^{n}, n \geq 3, \mathbb{R}^{n} \backslash \bar{\Omega} \neq \emptyset$ and let $w: \Omega \rightarrow[0, \infty]$ be a lower semicontinuous function such that $\operatorname{int}\{w=0\}=\emptyset$. We say that $w$ is a density with the mean value property for nonnegative harmonic functions in $\Omega$ if

(i) $w(\Omega):=\int_{\Omega} w(y) d y<\infty$,

(ii) there exists a point $x_{0} \in \Omega$ such that

$$
u\left(x_{0}\right)=\frac{1}{w(\Omega)} \int_{\Omega} u(y) w(y) d y
$$

for every harmonic function $u$ in $\Omega, u \geq 0$.

For the sake of simplicity, if $w$ is a density with the mean value property for the harmonic nonnegative functions in $\Omega$, we say that

$$
\left(\Omega, w, x_{0}\right) \text { is a } \Delta \text {-triple; }
$$

as usual $\Delta$ denotes the classical Laplace operator in $\mathbb{R}^{n}$.

A basic example of $\Delta$-triple is $\left(B_{r}\left(x_{0}\right), 1, x_{0}\right)$, where $B_{r}\left(x_{0}\right)$ is the Euclidean ball with center $x_{0}$ and radius $r$. Indeed, by the Gauss Theorem,

$$
u\left(x_{0}\right)=\frac{1}{\left|B_{r}\left(x_{0}\right)\right|} \int_{B_{r}\left(x_{0}\right)} u(y) d y, \quad \forall u \in \mathcal{H}\left(B_{r}\left(x_{0}\right)\right), u \geq 0,
$$

where $\left|B_{r}\left(x_{0}\right)\right|$ stands for the Lebesgue measure of $B_{r}\left(x_{0}\right)$ and $\mathcal{H}\left(B_{r}\left(x_{0}\right)\right)$ denotes the space of the harmonic functions in $B_{r}\left(x_{0}\right)$.

More general $\Delta$-triples can be obtained using the densities with the mean value property for harmonic functions constructed by Hansen-Netuka [10] and Aikawa [2], [3], see also [6]. In particular, for every bounded $C^{1, \epsilon}$-open set $\Omega$, and for every $x_{0} \in \Omega$, there exists a (non-unique) density $w$ such that $\left(\Omega, w, x_{0}\right)$ is a $\Delta$-triple.

2010 Mathematics Subject Classification. Primary: 31B05; Secondary: 35H20.

Key words and phrases. sub-Laplacian, mean value property, inverse problem.

Corresponding author: ermanno.lanconelli@unibo.it.

Acknowledgement: The first author has been supported by the Gruppo Nazionale per l'Analisi Matematica, la Probabilità e le loro Applicazioni (GNAMPA) of the Istituto Nazionale di Alta Matematica (INdAM). 
The problem of the best harmonic $L^{1}$-approximation of subharmonic functions, see [9], suggests the following inverse problem:

(IP) if $\left(\Omega, w, x_{0}\right)$ and $\left(D, w^{\prime}, x_{0}\right)$ are $\Delta$-triples, such that

$$
\frac{w}{w(\Omega)}=\frac{w^{\prime}}{w^{\prime}(D)} \quad \text { in } \Omega \cap D
$$

is it true that $\Omega=D$ ?

Positive answers to (IP), in the case $\Omega$ is an Euclidean ball, were given by Epstein [7], EpsteinSchiffer [8], Kuran [12]. In our language, their results can be stated as follows:

let $D \subset \mathbb{R}^{n}$ be an open connected set with finite Lebesgue measure. If $\left(D, 1, x_{0}\right)$ is a $\Delta$-triple and $r>0$ is such that $\frac{1}{|D|}=\frac{1}{\left|B_{r}\left(x_{0}\right)\right|}$, then $D=B_{r}\left(x_{0}\right)$.

Notice that the Euclidean balls play a privileged role here; indeed $\left(B_{r}\left(x_{0}\right), 1, x_{0}\right)$ is not only a $\Delta$-triple, as previously observed, but it has the following extra-property: if we denote by $\Gamma$ the fundamental solution with pole at 0 of the Laplace operator in $\mathbb{R}^{n}, n \geq 3$, then

$$
\Gamma\left(x_{0}-x\right)>\frac{1}{\left|B_{r}\left(x_{0}\right)\right|} \int_{B_{r}\left(x_{0}\right)} \Gamma(y-x) d y \quad \text { for all } x \in B_{r}\left(x_{0}\right) \backslash\left\{x_{0}\right\} .
$$

Moreover, the Euclidean balls also have some trivial, but important for our aims, topological properties: $B_{r}\left(x_{0}\right)=\operatorname{int} \overline{B_{r}\left(x_{0}\right)}$ and $\mathbb{R}^{n} \backslash \overline{B_{r}\left(x_{0}\right)}$ is connected.

These properties of the Euclidean balls lead us to give the following definition.

Definition 1.1. Let $\Omega$ be an open set in $\mathbb{R}^{n}, n \geq 3$. We say that $\left(\Omega, w, x_{0}\right)$ is a strong $\Delta$-triple if

(a) $\left(\Omega, w, x_{0}\right)$ is a $\Delta$-triple,

(b) $\Gamma\left(x_{0}-x\right)>\frac{1}{w(\Omega)} \int_{\Omega} \Gamma(y-x) w(y) d y$, for every $x \in \Omega \backslash\left\{x_{0}\right\}$.

Moreover, we say that $\Omega$ is solid, if $\Omega=\operatorname{int} \bar{\Omega}$ and $\mathbb{R}^{n} \backslash \bar{\Omega}$ is a connected, not empty set.

In the very recent paper [6], we proved a result (Theorem 1.1) implying, as a corollary, the following theorem.

Theorem 1.2. Let $\Omega, D$ be bounded, open sets in $\mathbb{R}^{n}, n \geq 3$. Assume that

(i) $\left(\Omega, w, x_{0}\right)$ is a strong $\Delta$-triple and $x \mapsto \int_{\mathbb{R}^{n}} \Gamma(y-x) w(y) d y$ is continuous,

(ii) $\left(D, w^{\prime}, x_{0}\right)$ is a $\Delta$-triple,

(iii) $\frac{w}{w(\Omega)}=\frac{w^{\prime}}{w^{\prime}(D)}$ in $\Omega \cap D$,

(iv) $\Omega$ is a solid set.

Then $D=\Omega$ and $w^{\prime}=\frac{w^{\prime}(D)}{w(\Omega)} w$.

In the present paper we will prove a more general version of [6, Theorem 1.1], see Theorem 3.4, so also obtaining a more general result than Theorem 1.2, see Theorem 3.1. Precisely, we will improve the results in [6] in two directions: the involved operators will be not only the classical Laplacian, but any sub-Laplacian on a stratified group; moreover, the boundedness assumptions on $\Omega$ and $D$, and the continuity assumption in (i) will be removed.

The plan of the paper is the following. In the next section, we will introduce the sub-Laplacian operators $\mathcal{L}$ and we will recall some of their fundamental properties. Moreover, we will give the definitions of $\mathcal{L}$-triples, strong $\mathcal{L}$-triples and, correspondingly, $\Gamma$-triples and strong $\Gamma$-triples, with $\Gamma$ 
the fundamental solution of $\mathcal{L}$. We will also exhibit examples of strong $\mathcal{L}$-triples, see Theorem 2.4. In Section 3 we will state our results on the inverse problem (Theorem 3.1, Corollary 3.2, Theorem 3.4 and Corollary 3.5) and in Section 4 we will prove them. In the Appendix, Section 5, for reader's convenience, we will recall the definition and list some properties of the $\mathcal{L}$-superharmonic functions as presented in [5, Chapter 8].

\section{Sub-Laplacians AND RELATEd TRIPLES}

A sum of squares operator

$$
\mathcal{L}=\sum_{i=1}^{m} X_{j}^{2},
$$

is a sub-Laplacian in $\mathbb{R}^{n}$ if the following conditions hold.

(H1) The $X_{j}$ 's are smooth vector fields in $\mathbb{R}^{n}$ and generate a Lie algebra $\mathfrak{a}$ satisfying rank $\mathfrak{a}(x)=$ $\operatorname{dim} \mathfrak{a}=n$ at any point $x \in \mathbb{R}^{n}$.

(H2) There exists a group of dilations $\left(\delta_{\lambda}\right)_{\lambda>0}$ in $\mathbb{R}^{n}$ such that every vector field $X_{j}$ is $\delta_{\lambda^{-}}$ homogeneous of degree one.

A group of dilations in $\mathbb{R}^{n}$ is a family of diagonal linear functions $\left(\delta_{\lambda}\right)_{\lambda>0}$ of the kind

$$
\delta_{\lambda}\left(x_{1}, \ldots, x_{n}\right)=\left(\lambda^{\sigma_{1}} x_{1}, \ldots, \lambda^{\sigma_{n}} x_{n}\right),
$$

where the $\sigma_{j}$ 's are natural numbers.

Due to the rank condition in (H1), the operator $\mathcal{L}$ is hypoelliptic, see [11], so that the $\mathcal{L}$-harmonic functions, i.e., the solutions to $\mathcal{L} u=0$, are smooth.

Conditions (H1) and (H2) imply the existence of a group law $\circ$ making $\mathbb{G}=\left(\mathbb{R}^{n}, \circ, \delta_{\lambda}\right)$ a stratified Lie group on which every vector field $X_{j}$ is left translation invariant, see [4]. The natural number $Q:=\sigma_{1}+\ldots+\sigma_{N}$ is called the homogeneous dimension of $\mathbb{G}$. If $Q=2, \mathbb{G}$ is the Euclidean group and $\mathcal{L}$, up to a linear transformation, is the usual Laplace operator. From now on, we assume, without further comment, that $Q \geq 3$.

One of the main features of a sub-Laplacian $\mathcal{L}$ is the existence of a gauge function playing for it the same role played by the Euclidean norm for the classical Laplace operator. A $\mathcal{L}$-gauge is a continuous function $d: \mathbb{G} \rightarrow\left[0, \infty\left[\right.\right.$, $\mathbb{G}$-symmetric, i.e., $d\left(x^{-1}\right)=d(x)$ for every $x \in \mathbb{G}$, strictly positive and smooth outside the origin, which is $\delta_{\lambda}$-homogeneous of degree one, and such that

is $\mathcal{L}$-harmonic in $\mathbb{G} \backslash\{0\}$.

$$
\gamma(x):=\frac{1}{d(x)^{Q-2}}
$$

The $d$-balls $B_{r}^{d}(x):=\left\{y \in \mathbb{G}: d\left(x^{-1} \circ y\right)<r\right\}$ support averaging operators which characterize the $\mathcal{L}$-harmonic functions the same way as the usual mean value operators on Euclidean balls characterize classical harmonic functions. To be precise, define in $\mathbb{G} \backslash\{0\}$

$$
\psi:=\left|\nabla_{\mathcal{L}} d\right|^{2}, \quad \nabla_{\mathcal{L}}:=\left(X_{1}, \ldots, X_{m}\right),
$$

and

where

$$
M_{r}(u)(x):=\frac{m_{d}}{r^{Q}} \int_{B_{r}^{d}(x)} u(y) \psi\left(x^{-1} \circ y\right) d y,
$$

and

$$
m_{d}:=Q(Q-2) \beta_{d}
$$

$$
\left(\beta_{d}\right)^{-1}:=Q(Q-2) \int_{B_{1}^{d}(0)} \psi(y) d y .
$$


Then a continuous function $u: O \rightarrow \mathbb{R}, O \subseteq \mathbb{G}$ open, is smooth and satisfies $\mathcal{L} u=0$ in $O$ if and only if

$$
u(x)=M_{r}(u)(x) \quad \forall B_{r}^{d}(x) \subseteq O .
$$

This is Gauss-Koebe's Theorem for $\mathcal{L}$, see [5, Theorem 5.6.3].

The fundamental solution of $\mathcal{L}$ with pole at the origin is

$$
\Gamma:=\beta_{d} \gamma,
$$

see [5, Theorem 5.5.6].

We now give some definitions: $\mathcal{L}$-triples, strong $\mathcal{L}$-triples and, correspondingly, $\Gamma$-triples and strong $\Gamma$-triples.

Definition 2.1. Let $\Omega$ be an open subset of $\mathbb{G}$, such that $\mathbb{G} \backslash \bar{\Omega} \neq \emptyset$ and let $w: \Omega \rightarrow[0, \infty]$ be a lower semicontinuous function with $\operatorname{int}\{w=0\}=\emptyset$.

We say that $\left(\Omega, w, x_{0}\right)$ is a $\mathcal{L}$-triple if

(i) $w(\Omega):=\int_{\Omega} w(y) d y<\infty$,

(ii) there exists $x_{0} \in \Omega$ such that

$$
u\left(x_{0}\right)=\frac{1}{w(\Omega)} \int_{\Omega} u(y) w(y) d y
$$

for every $\mathcal{L}$-harmonic function $u$ in $\Omega, u \geq 0$.

If, moreover,

(iii) $\Gamma\left(x^{-1} \circ x_{0}\right)>\frac{1}{w(\Omega)} \int_{\Omega} \Gamma\left(x^{-1} \circ y\right) w(y) d y$, for every $x \in \Omega \backslash\left\{x_{0}\right\}$,

then we say that $\left(\Omega, w, x_{0}\right)$ is a strong $\mathcal{L}$-triple.

A variant of the notion of $\mathcal{L}$-triple is the following definition of $\Gamma$-triple. Before stating it, we recall that if $\Omega$ is an open subset of $\mathbb{G}, \mu$ is a nonnegative Radon measure in $\mathbb{G}, \mu\left(\Omega^{c}\right)=0$, then the $\Gamma$-potential of $\mu$ is defined as follows:

$$
\Gamma_{\mu}(x):=\int_{\Omega} \Gamma\left(x^{-1} \circ y\right) d \mu(y) \quad x \in \mathbb{G} .
$$

Definition 2.2. Let $\Omega$ be an open subset of $\mathbb{G}, x_{0}$ a point of $\Omega$ and let $\mu$ be a nonnegative Radon measure in $\mathbb{G}, \mu(\Omega)=1$ and $\mu\left(\Omega^{c}\right)=0$.

We say that $\left(\Omega, \mu, x_{0}\right)$ is a $\Gamma$-triple if

$$
\Gamma_{\mu}(x)=\Gamma\left(x^{-1} \circ x_{0}\right) \quad \forall x \in \Omega^{c} .
$$

If, moreover,

$$
\Gamma_{\mu}(x)<\Gamma\left(x^{-1} \circ x_{0}\right) \quad \forall x \in \Omega \backslash\left\{x_{0}\right\},
$$

then we say that $\left(\Omega, \mu, x_{0}\right)$ is a strong $\Gamma$-triple.

Remark 2.3. Let $\left(\Omega, w, x_{0}\right)$ be a $\mathcal{L}$-triple. Extend $w$ to $\mathbb{G}$ by letting $w$ be 0 in $\Omega^{c}$ and define $\mu$ the measure

$$
d \mu(y)=\frac{w(y)}{w(\Omega)} d y .
$$

Then $\left(\Omega, \mu, x_{0}\right)$ is a $\Gamma$-triple. Indeed, $\mu(\Omega)=1$ and, fixed $x \in \Omega^{c}$, (ii) in Definition 2.1, applied with $u(y):=\Gamma\left(x^{-1} \circ y\right)$, implies (2.5) in Definition 2.2. 
We stress that the present definition in the case of $\mathcal{L}=\Delta$, the classical Laplacian, is more general that the one given in [6]: indeed, we don't require anymore the boundedness of $\Omega$ and the continuity of $\Gamma_{\mu}$.

The Gauss-type Theorem for sub-Laplacians recalled above implies that $\left(B_{r}^{d}\left(x_{0}\right), \psi\left(x_{0}^{-1} \circ \cdot\right), x_{0}\right)$ is a $\mathcal{L}$-triple. Actually, it is a strong $\mathcal{L}$-triple; as a matter of fact, more general strong $\mathcal{L}$-triples can be defined on every $d$-ball, as the following theorem shows.

Theorem 2.4. Let $f:] 0, \infty[\rightarrow] 0, \infty[$ be a continuous function, such that

$$
\left.F(r):=\int_{0}^{r} f(\rho) d \rho<\int_{0}^{\infty} f(\rho) d \rho=\infty \quad \forall r \in\right] 0, \infty[.
$$

Define

$$
w_{f}(y):=\frac{f(d(y))}{d(y)^{Q-1}} \psi(y), \quad y \in \mathbb{G} \backslash\{0\},
$$

where $\psi$ is the function in (2.2).

Then $\operatorname{int}\left\{w_{f}=0\right\}=\emptyset$ and, for every $x_{0} \in \mathbb{G}$ and $r>0$,

$$
\left(B_{r}^{d}\left(x_{0}\right), w_{f}\left(x_{0}^{-1} \circ \cdot\right), x_{0}\right)
$$

is a strong $\mathcal{L}$-triple.

We agree to say that the function

$$
y \mapsto w_{f}\left(x_{0}^{-1} \circ \cdot\right) \quad y \in \mathbb{G} \backslash\left\{x_{0}\right\},
$$

with $w_{f}$ defined in $(2.7)$, is $\mathcal{L}$-radially symmetric with respect to $x_{0}$.

Proof of Theorem 2.4. We first observe that $\left\{w_{f}=0\right\}=\{\psi=0\}$ and this last set has empty interior as proved in [5, page 262].

Let us now prove that $\left(B_{r}^{d}\left(x_{0}\right), w_{f}\left(x_{0}^{-1} \circ \cdot\right), x_{0}\right)$ is a strong $\mathcal{L}$-triple. The proof relies on $[5$, Theorem 9.5.2] and the coarea formula.

Let us first prove that $\left(B_{r}^{d}\left(x_{0}\right), w_{f}\left(x_{0}^{-1} \circ \cdot\right), x_{0}\right)$ is a $\mathcal{L}$-triple.

By formula [5, (9.22)], for every $\mathcal{L}$-harmonic nonnegative function $u$ in $B_{r}^{d}\left(x_{0}\right)$ and for every $\rho<r$,

$$
u\left(x_{0}\right)=\mathcal{M}_{\rho}(u)\left(x_{0}\right),
$$

where $\mathcal{M}_{\rho}$ is the surface average operator defined in $[5,(5.46)]$; i.e.,

$$
\mathcal{M}_{\rho}(u)\left(x_{0}\right):=\frac{(Q-2) \beta_{d}}{\rho^{Q-1}} \int_{\partial B_{\rho}^{d}\left(x_{0}\right)} u(y) \frac{\psi\left(x_{0}^{-1} \circ y\right)}{\left|\nabla d\left(x_{0}^{-1} \circ y\right)\right|} d \sigma(y),
$$

with $\beta_{d}$ as in (2.4).

Let us multiply (2.8) by $\frac{f(\rho)}{F(r)}$ and integrate w.r.t. $\rho$ on $] 0, r[$. By the coarea formula we get

$$
\begin{aligned}
u\left(x_{0}\right) & =\frac{1}{F(r)} \int_{0}^{r} f(\rho) \mathcal{M}_{\rho}(u)\left(x_{0}\right) d \rho \\
& =\frac{(Q-2) \beta_{d}}{F(r)} \int_{0}^{r}\left(\int_{\partial B_{\rho}^{d}\left(x_{0}\right)} u(y) \frac{f\left(d\left(x_{0}^{-1} \circ y\right)\right)}{d\left(x_{0}^{-1} \circ y\right)^{Q-1}} \psi\left(x_{0}^{-1} \circ y\right) \frac{d \sigma(y)}{\left|\nabla d\left(x_{0}^{-1} \circ y\right)\right|}\right) d \rho \\
& =\frac{(Q-2) \beta_{d}}{F(r)} \int_{B_{r}^{d}\left(x_{0}\right)} u(y) w_{f}\left(x_{0}^{-1} \circ y\right) d y .
\end{aligned}
$$


If we take $u=1$ in the previous identities, we obtain

i.e.,

$$
1=\frac{(Q-2) \beta_{d}}{F(r)} \int_{B_{r}^{d}\left(x_{0}\right)} w_{f}\left(x_{0}^{-1} \circ y\right) d y
$$

$$
w_{f}\left(x_{0}^{-1} \circ B_{r}^{d}\left(x_{0}\right)\right):=\frac{F(r)}{(Q-2) \beta_{d}}<\infty .
$$

Therefore, $\left(B_{r}^{d}\left(x_{0}\right), w_{f}\left(x_{0}^{-1} \circ \cdot\right), x_{0}\right)$ is a $\mathcal{L}$-triple.

To show that this triple is strong, we only need to prove that $w_{f}$ satisfies (iii) in Definition 2.1.

For every $x \in \mathbb{G}$ let us define

$$
u_{x}(y):=\Gamma\left(x^{-1} \circ y\right) \quad y \in \mathbb{G} .
$$

We remark that $u_{x}$ is a $\mathcal{L}$-superharmonic function and

$$
\mathcal{L} u_{x}=-\delta_{x} \quad \text { in the sense of distributions, }
$$

where $\delta_{x}$ is the Dirac measure at $\{x\}$. By Poisson-Jensen's formula [5, Theorem 9.5.2], for every $\rho>0$,

$$
u_{x}\left(x_{0}\right)=\mathcal{M}_{\rho}\left(u_{x}\right)\left(x_{0}\right)+\int_{B_{\rho}^{d}\left(x_{0}\right)}\left(\Gamma\left(x_{0}^{-1} \circ y\right)-\Gamma(\rho)\right) d \delta_{x}(y),
$$

where $\mathcal{M}_{\rho}$ is the surface average operator in (2.9). We have

$$
\int_{B_{\rho}^{d}\left(x_{0}\right)}\left(\Gamma\left(x_{0}^{-1} \circ y\right)-\Gamma(\rho)\right) d \delta_{x}(y)= \begin{cases}0 & \text { if } d\left(x_{0}^{-1} \circ x\right) \geq \rho \\ \Gamma\left(x_{0}^{-1} \circ x\right)-\Gamma(\rho) & \text { if } 0<d\left(x_{0}^{-1} \circ x\right)<\rho .\end{cases}
$$

Therefore, (2.12) and the equality $\Gamma\left(x^{-1} \circ x_{0}\right)=\Gamma\left(x_{0}^{-1} \circ x\right)$, give

$$
\mathcal{M}_{\rho}\left(u_{x}\right)\left(x_{0}\right)= \begin{cases}u_{x}\left(x_{0}\right)=\Gamma\left(x^{-1} \circ x_{0}\right) & \text { if } d\left(x_{0}^{-1} \circ x\right) \geq \rho \\ \Gamma(\rho)<\Gamma\left(x_{0}^{-1} \circ x\right) & \text { if } 0<d\left(x_{0}^{-1} \circ x\right)<\rho .\end{cases}
$$

Let us now consider $x \in B_{r}^{d}\left(x_{0}\right) \backslash\left\{x_{0}\right\}$.

By (2.10), (2.13) and (2.11),

$$
\begin{aligned}
& \int_{B_{r}^{d}\left(x_{0}\right)} \Gamma\left(x^{-1} \circ y\right) w_{f}\left(x_{0}^{-1} \circ y\right) d y=\frac{1}{(Q-2) \beta_{d}} \int_{0}^{r} f(\rho) \mathcal{M}_{\rho}\left(u_{x}\right)\left(x_{0}\right) d \rho \\
& =\int_{0}^{d\left(x_{0}^{-1} \circ x\right)} \frac{f(\rho)}{(Q-2) \beta_{d}} \mathcal{M}_{\rho}\left(u_{x}\right)\left(x_{0}\right) d \rho+\int_{d\left(x_{0}^{-1} \circ x\right)}^{r} \frac{f(\rho)}{(Q-2) \beta_{d}} \mathcal{M}_{\rho}\left(u_{x}\right)\left(x_{0}\right) d \rho \\
& <\Gamma\left(x^{-1} \circ x_{0}\right) \frac{1}{(Q-2) \beta_{d}} \int_{0}^{r} f(\rho) d \rho=\Gamma\left(x^{-1} \circ x_{0}\right) w_{f}\left(x_{0}^{-1} \circ B_{r}^{d}\left(x_{0}\right)\right) .
\end{aligned}
$$

This concludes the proof.

\section{Results ON The inVERSE PROBlem FOR $\mathcal{L}$}

In this section we state and prove our main results regarding the analogue of the inverse problem (IP) for the sub-Laplacian, see Theorems 3.1 and 3.4. As an application, we show that the $d$-balls are the only open sets supporting $\mathcal{L}$-radially symmetric densities with the mean value property for $\mathcal{L}$, see Corollary 3.2 and the related Corollary 3.5.

Theorem 3.1. Let $\Omega, D$ be open sets in $\mathbb{G}$, such that $(\bar{\Omega} \cup \bar{D})^{c} \neq \emptyset$.

Assume that

(i) $\left(\Omega, w, x_{0}\right)$ is a strong $\mathcal{L}$-triple, 
(ii) $\left(D, w^{\prime}, x_{0}\right)$ is a $\mathcal{L}$-triple,

(iii) $\frac{w}{w(\Omega)}=\frac{w^{\prime}}{w^{\prime}(D)}$ in $\Omega \cap D$,

(iv) $\Omega$ is a solid set.

Then $D=\Omega$ and $w^{\prime}=\frac{w^{\prime}(D)}{w(\Omega)} w$.

If we apply this theorem to the strong $\mathcal{L}$-triples given by Theorem 2.4, we obtain the following $\mathcal{L}$-harmonic characterization of the $d$-balls.

Corollary 3.2. Let $D$ be an open set in $\mathbb{G}$ such that $\bar{D}^{c}$ is unbounded. Let $w_{f}$ be the $\mathcal{L}$-radially symmetric function in (2.7) and assume that, for some $x_{0} \in D$,

(a) $w_{f}\left(x_{0}^{-1} \circ D\right)<\infty$,

(b) $u\left(x_{0}\right)=\frac{1}{w_{f}\left(x_{0}^{-1} \circ D\right)} \int_{D} u(y) w_{f}\left(x_{0}^{-1} \circ y\right) d y$ for every nonnegative $\mathcal{L}$-harmonic function in $D$.

Then

$$
D=B_{r}^{d}\left(x_{0}\right)
$$

where $r$ is the only real positive number such that

$$
w_{f}\left(x_{0}^{-1} \circ D\right)=w_{f}\left(x_{0}^{-1} \circ B_{r}^{d}\left(x_{0}\right)\right) .
$$

Remark 3.3. The unique number $r$ such that the equality (3.1) holds is, by (2.11), the only real positive number such that

$$
\int_{0}^{r} f(\rho) d \rho=(Q-2) \beta_{d} w_{f}\left(x_{0}^{-1} \circ D\right) .
$$

In the particular case of $f(\rho)=\rho^{Q-1}$ and $D$ a bounded set, Corollary 3.2 is Theorem 1.1 in [13]. Moreover, if $\mathbb{G}$ is the Euclidean group $\mathbb{R}^{n}, d$ is the Euclidean norm, $\mathcal{L}$ is the classical Laplace operator and $D$ is a connected set with finite Lebesgue measure, then Corollary 3.2 was proved in [12].

Theorem 3.1 above is a consequence of a result on $\Gamma$-triples, that, even in the case of the Laplacian, is more general than the analogue [6, Theorem 1.1].

Before stating it, we recall that the support of a measure $\mu$ can be defined as follows:

$$
\text { supp } \mu:=\{x \in \mathbb{G}:(A \text { open set, } x \in A) \Rightarrow \mu(A)>0\} \text {. }
$$

Theorem 3.4. Let $\Omega$ and $D$ be open sets in $\mathbb{G}$ containing $x_{0},(\bar{\Omega} \cup \bar{D})^{c} \neq \emptyset$.

Assume that

(i) $\left(\Omega, \mu, x_{0}\right)$ is a strong $\Gamma$-triple,

(ii) $\left(D, \nu, x_{0}\right)$ is a $\Gamma$-triple,

(iii) $\mu\llcorner(\Omega \cap D)=\nu\llcorner(\Omega \cap D)$,

(iv) $\partial D \subseteq \operatorname{supp} \nu$,

(v) $\Omega$ is a solid set.

Then $D=\Omega$ and $\nu=\mu$.

Examples given in [6] for the Laplace operator show that the assumptions are essentially sharp: the request that $\left(\Omega, \mu, x_{0}\right)$ is a strong $\Gamma$-triple cannot be weakened by assuming that $\left(\Omega, \mu, x_{0}\right)$ is simply a $\Gamma$-triple and neither (iii) nor (iv) can be removed.

If we apply this theorem to the strong $\Gamma$-triples given by Theorem 2.4 and Remark 2.3 we obtain the following characterization of the $d$-balls. 
Corollary 3.5. Let $D$ be an open set in $\mathbb{G}$ such that $\bar{D}^{c}$ is unbounded. Let $w_{f}$ be the $\mathcal{L}$-radially symmetric function in (2.7) and assume that, for some $x_{0} \in D$,

(a) $w_{f}\left(x_{0}^{-1} \circ D\right)<\infty$,

Then

(b) $\Gamma\left(x^{-1} \circ x_{0}\right)=\frac{1}{w_{f}\left(x_{0}^{-1} \circ D\right)} \int_{D} \Gamma\left(x^{-1} \circ y\right) w_{f}\left(x_{0}^{-1} \circ y\right) d y$ for every $x \notin D$.

where $r$ is the only real positive number such that

$$
D=B_{r}^{d}\left(x_{0}\right)
$$

$$
w_{f}\left(x_{0}^{-1} \circ D\right)=w_{f}\left(x_{0}^{-1} \circ B_{r}^{d}\left(x_{0}\right)\right) .
$$

In the particular case of $f(\rho)=\rho^{Q-1}$ and $D$ is a bounded set, Corollary 3.5 is Theorem $1.2 \mathrm{in}$ [13]. Moreover, if $\mathbb{G}$ is the Euclidean group $\mathbb{R}^{n}, d$ is the Euclidean norm, $\mathcal{L}$ is the classical Laplace operator and $D$ is bounded, then Corollary 3.5 is a result by Aharonov-Schiffer-Zalcman in [1].

\section{Proofs of the Results on the InVERSE Problem}

In this section we prove the results stated in Section 3.

Proof of Theorem 3.4. We split the proof in four steps.

STEP 1. Let us prove that $\Gamma_{\mu} \leq \Gamma_{\nu}$ in $\mathbb{G} \backslash\left\{x_{0}\right\}$.

Assumption (i) and (ii) imply

$$
\Gamma_{\mu}(x) \leq \Gamma\left(x^{-1} \circ x_{0}\right)<\infty \quad \forall x \in \mathbb{G} \backslash\left\{x_{0}\right\}, \quad \Gamma_{\nu}(x)=\Gamma\left(x^{-1} \circ x_{0}\right) \quad \forall x \in D^{c} .
$$

Then, since $x_{0} \notin D^{c}$,

$$
\Gamma_{\mu}(x) \leq \Gamma_{\nu}(x) \quad \forall x \in D^{c} .
$$

It remains to prove that $\Gamma_{\mu} \leq \Gamma_{\nu}$ in $D \backslash\left\{x_{0}\right\}$.

We first remark that, by the first chain of inequalities in (4.1),

$$
\Gamma_{\mu}-\Gamma_{\nu} \text { is well defined and }<\infty \text { in } D \backslash\left\{x_{0}\right\} .
$$

Moreover, by using (iii), one easily recognizes that

$$
\Gamma_{\mu}(x)-\Gamma_{\nu}(x)=\int_{\Omega \backslash D} \Gamma\left(x^{-1} \circ y\right) d \mu(y)-\int_{D \backslash \Omega} \Gamma\left(x^{-1} \circ y\right) d \nu(y) \quad \forall x \in D \backslash\left\{x_{0}\right\} .
$$

Hereafter we agree to let an integral be equal to zero, if the integration domain is empty.

The functions

$$
h(x):=\int_{\Omega \backslash D} \Gamma\left(x^{-1} \circ y\right) d \mu(y), \quad x \in D
$$

and

$$
v(x):=\int_{D \backslash \Omega} \Gamma\left(x^{-1} \circ y\right) d \nu(y), \quad x \in D
$$

are, respectively, $\mathcal{L}$-harmonic and $\mathcal{L}$-superharmonic in $D$, see Section 5 . As a consequence,

$$
\tilde{u}:=h-v \text { is } \mathcal{L} \text {-subharmonic in } D ;
$$

moreover, keeping in mind (4.2),

$$
\tilde{u}=\Gamma_{\mu}-\Gamma_{\nu} \quad \text { in } D \backslash\left\{x_{0}\right\} .
$$

On the other hand, by the first item in (4.1) and the lower semicontinuity of $\Gamma_{\nu}$, for every $x \in \partial D$,

$$
\limsup _{D \ni y \rightarrow x} \tilde{u}(y)=\limsup _{D \ni y \rightarrow x}\left(\Gamma_{\mu}-\Gamma_{\nu}\right)(y) \leq \limsup _{D \ni y \rightarrow x}\left(\Gamma\left(y^{-1} \circ x_{0}\right)-\Gamma_{\nu}(y)\right)
$$




$$
\leq \Gamma\left(x^{-1} \circ x_{0}\right)-\Gamma_{\nu}(x)=0,
$$

since $\left(D, \nu, x_{0}\right)$ is a $\Gamma$-triple and $x \notin D$. Moreover,

$$
\limsup _{D \ni y \rightarrow \infty} \tilde{u}(y) \leq \limsup _{D \ni y \rightarrow \infty}\left(\Gamma\left(y^{-1} \circ x_{0}\right)-\Gamma_{\nu}(y)\right) \leq \limsup _{y \rightarrow \infty} \Gamma\left(y^{-1} \circ x_{0}\right)=0 .
$$

By the maximum principle for subharmonic functions (see [5, Theorem 8.2.19 (ii)]) we get $\tilde{u} \leq 0$ in $D$; hence $\Gamma_{\mu} \leq \Gamma_{\nu}$ in $D \backslash\left\{x_{0}\right\}$.

STEP 2. Let us prove that $\partial D \subseteq \bar{\Omega}$.

By contradiction, assume there exists a point $x \in \partial D$ such that $x \notin \bar{\Omega}$. Then $x \in \operatorname{supp} \nu$ (by assumption (iv)) and $\mathbb{G} \backslash \bar{\Omega}$ is an open set containing $x$. As a consequence

$$
\nu(\mathbb{G} \backslash \bar{\Omega})>0 .
$$

Since $\mu$ has its support contained in $\bar{\Omega}, \Gamma_{\mu}$ is $\mathcal{L}$-harmonic in $\mathbb{G} \backslash \bar{\Omega}$, see Section 5 , so that

$$
\Gamma_{\mu}-\Gamma_{\nu} \text { is } \mathcal{L} \text {-subharmonic in } \mathbb{G} \backslash \bar{\Omega} \text {. }
$$

On the other hand, by what we proved in Step $1, \Gamma_{\mu}-\Gamma_{\nu} \leq 0$ in $\mathbb{G} \backslash \bar{\Omega}$. Moreover, since (i) and (ii) imply

$$
\Gamma_{\mu}=\Gamma_{\nu} \quad \text { in } \Omega^{c} \cap D^{c}
$$

then $\left(\Gamma_{\mu}-\Gamma_{\nu}\right)(x)=0$,

By (v) $\mathbb{G} \backslash \bar{\Omega}$ is a connected set, so, the strong maximum principle for subharmonic functions (see in $[5$, Theorem 8.2.19 (i)]) imply

$$
\Gamma_{\mu}-\Gamma_{\nu}=0 \quad \text { in } \mathbb{G} \backslash \bar{\Omega},
$$

so that

$$
\mathcal{L}\left(\Gamma_{\mu}-\Gamma_{\nu}\right)=0 \quad \text { in } \mathbb{G} \backslash \bar{\Omega} .
$$

On the other hand, in $\mathbb{G} \backslash \bar{\Omega}, \mathcal{L}\left(\Gamma_{\mu}-\Gamma_{\nu}\right)=\nu$. Therefore, $\nu(\mathbb{G} \backslash \bar{\Omega})=0$, in contradiction with (4.3).

STEP 3. Let us prove that $D \subseteq \Omega$.

We have

$$
\mathbb{G} \backslash \bar{\Omega}=\left(D \cup D^{c}\right) \backslash \bar{\Omega}=(D \backslash \bar{\Omega}) \cup(\partial D \backslash \bar{\Omega}) \cup\left(\bar{D}^{c} \cap \bar{\Omega}^{c}\right)=(D \backslash \bar{\Omega}) \cup(\bar{D} \cup \bar{\Omega})^{c} .
$$

By assumption, $(\bar{D} \cup \bar{\Omega})^{c}$ is not empty. Moreover, $D \backslash \bar{\Omega}$ and $(\bar{D} \cup \bar{\Omega})^{c}$ are open, disjoint sets. The set $\mathbb{G} \backslash \bar{\Omega}$ is connected by (v), then $D \backslash \bar{\Omega}$ must be empty. Therefore $D \subseteq \bar{\Omega}$. By (v) we have that $\operatorname{int} \bar{\Omega}=\Omega$, thus we obtain $D \subseteq \Omega$.

STEP 4. Let us prove that $\Omega \subseteq D$. We argue by contradiction; i.,e, we assume that there exists

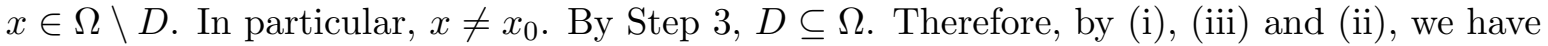

$$
\begin{aligned}
\Gamma\left(x^{-1} \circ x_{0}\right) & >\Gamma_{\mu}(x)=\int_{D} \Gamma\left(x^{-1} \circ y\right) d \mu(y)+\int_{\Omega \backslash D} \Gamma\left(x^{-1} \circ y\right) d \mu(y) \\
& \geq \int_{D} \Gamma\left(x^{-1} \circ y\right) d \mu(y)=\int_{D} \Gamma\left(x^{-1} \circ y\right) d \nu(y)=\Gamma_{\nu}(x)=\Gamma\left(x^{-1} \circ x_{0}\right) .
\end{aligned}
$$

This is an absurd.

We have so proved that $D=\Omega$ and, consequently, that $\mu=\nu$.

As a corollary of Theorem 3.4, we get Theorem 3.1 
Proof of Theorem 3.1. Let us extend $w$ and $w^{\prime}$ with 0 to all $\mathbb{G}$ and define the measures $\mu, \nu$ as follows:

$$
d \mu(y)=\frac{w(y)}{w(\Omega)} d y, \quad d \nu(y)=\frac{w^{\prime}(y)}{w^{\prime}(D)} d y .
$$

By (i), (ii) and Remark 2.3, $\left(D, \nu, x_{0}\right)$ is a $\Gamma$-triple and $\left(\Omega, \mu, x_{0}\right)$ is a strong $\Gamma$-triple. By (iii), $\mu\left\llcorner(\Omega \cap D)=\nu\left\llcorner(\Omega \cap D)\right.\right.$. Moreover, by definition of $\mathcal{L}$-triple, since $\operatorname{int}\left\{y \in D: w^{\prime}(y)=0\right\}=\emptyset$, then $\partial D \subseteq \operatorname{supp} \nu$. The conclusion follows by Theorem 3.4.

We now are ready to prove Corollary 3.2.

Proof of Corollary 3.2. In order to apply Theorem 3.1 it is convenient to introduce the following notation:

$$
\begin{aligned}
& \Omega:=B_{r}^{d}\left(x_{0}\right) \quad \text { with } r>0 \text { given by }(3.2), \\
& w(y):=w_{f}\left(x_{0}^{-1} \circ y\right), \quad y \in \Omega, \\
& w^{\prime}(y):=w_{f}\left(x_{0}^{-1} \circ y\right) \quad y \in D .
\end{aligned}
$$

Since $\bar{D}^{c}$ is unbounded, then $(\bar{\Omega} \cup \bar{D})^{c} \neq \emptyset$. Moreover,

(i) $\left(\Omega, w, x_{0}\right)$ is a strong $\mathcal{L}$-triple (by Theorem 2.4$)$

(ii) $\left(D, w^{\prime}, x_{0}\right)$ is a $\mathcal{L}$-triple (by hypotheses (a) and (b)),

(iii) $\frac{w}{w(\Omega)}=\frac{w^{\prime}}{w^{\prime}(D)}$ in $\Omega \cap D\left(\right.$ since $w=w^{\prime}$ in $\Omega \cap D$ and, by (3.1), $\left.w(\Omega)=w^{\prime}(D)\right)$,

(iv) $\Omega$ is a solid set.

As far as (iv) is concerned, it is quite obvious that $B_{r}^{d}\left(x_{0}\right)=\operatorname{int} \overline{B_{r}^{d}\left(x_{0}\right)}$; the second condition, $\mathbb{G} \backslash \overline{B_{r}^{d}\left(x_{0}\right)}$ is connected, can be proved as follows.

Let $B$ be an Euclidean ball containing $\overline{B_{r}^{d}\left(x_{0}\right)}$. Then for every $x, y \in \mathbb{G} \backslash \overline{B_{r}^{d}\left(x_{0}\right)}$,

$$
(\mathbb{G} \backslash B) \cup\left\{\delta_{\lambda}(x): \lambda \geq 1\right\} \cup\left\{\delta_{\lambda}(y): \lambda \geq 1\right\}
$$

is a connected subset in $\mathbb{G} \backslash \overline{B_{r}^{d}\left(x_{0}\right)}$ and it contains $x$ and $y$.

Then, all the assumptions of Theorem 3.1 are satisfied; hence $D=\Omega$; i.e.,

$$
D=B_{r}^{d}\left(x_{0}\right) \text {. }
$$

We now turn to the proof of Corollary 3.5.

Proof of Corollary 3.5. Define

$$
d \nu(y):=\frac{w_{f}\left(x_{0}^{-1} \circ y\right)}{w_{f}\left(x_{0}^{-1} \circ D\right)} \chi_{D}(y) d y .
$$

By (a) and (b) $\left(D, \nu, x_{0}\right)$ is a $\Gamma$-triple. Since by Theorem $2.4 \operatorname{int}\left\{y \in D: w_{f}\left(x_{0}^{-1} \circ y\right)=0\right\}=\emptyset$, then $\partial D \subseteq \bar{D}=\operatorname{supp}(\nu)$.

Let us choose $r>0$ such that (3.3) holds and define

$$
d \mu(y):=\frac{w_{f}\left(x_{0}^{-1} \circ y\right)}{w_{f}\left(x_{0}^{-1} \circ B_{r}^{d}\left(x_{0}\right)\right)} \chi_{B_{r}^{d}\left(x_{0}\right)}(y) d y .
$$

In particular, (iii) in Theorem 3.4 holds.

By Theorem $2.4\left(B_{r}^{d}\left(x_{0}\right), w_{f}\left(x_{0}^{-1} \circ \cdot\right), x_{0}\right)$ is a strong $\mathcal{L}$-triple, therefore, by Remark $2.3,\left(B_{r}^{d}\left(x_{0}\right), \mu, x_{0}\right)$ is a strong $\Gamma$-triple. Taking also into account that $B_{r}^{d}\left(x_{0}\right)$ is a solid set (see the proof of Corollary 
3.2) we have that all the assumptions of Theorem 3.4 are satisfied with $\Omega=B_{r}^{d}\left(x_{0}\right)$. The conclusion follows.

\section{Appendix: $\mathcal{L}$-superharmonic functions}

In this section we recall the definition and list some properties of the $\mathcal{L}$-superharmonic functions, as presented in [5, Chapter 8].

Let $\Omega \subseteq \mathbb{G}$ be open and let $u: \Omega \rightarrow]-\infty, \infty]$ be lower semicontinuous. We say that $u$ is $\mathcal{L}$-superharmonic in $\Omega$ if

(a) $u \in L_{\text {loc }}^{1}(\Omega)$ and $\mathcal{L}(u) \leq 0$ in $\Omega$ in the weak sense of distributions,

(b) $u$ is $M_{r}$-continuous; i.e.,

$$
u(x)=\lim _{r \rightarrow 0^{+}} M_{r}(u)(x) \quad \forall x \in \Omega .
$$

Here $M_{r}$ denotes the average operator in (2.3).

A function $v: \Omega \rightarrow[-\infty, \infty[$ is $\mathcal{L}$-subharmonic if $-v$ is $\mathcal{L}$-superharmonic. We say that $v$ is $\mathcal{L}$-harmonic if $v$ is smooth and $\mathcal{L} v=0$.

Let $\Gamma$ be the fundamental solution of $\mathcal{L}$ and let $\mu$ be a nonnegative Radon measure in $\mathbb{G}$. The $\Gamma$-potential of $\mu$ is defined as follows

$$
\Gamma_{\mu}(x):=\int_{\mathbb{G}} \Gamma\left(x^{-1} \circ y\right) d \mu(y), \quad x \in \mathbb{G} .
$$

Obviously, if $\Omega$ is an open set such that $\mu\left(\Omega^{c}\right)=0$,

$$
\Gamma_{\mu}(x)=\int_{\Omega} \Gamma\left(x^{-1} \circ y\right) d \mu(y), \quad x \in \Omega .
$$

The function $\Gamma_{\mu}$ is nonnegative and lower semicontinuous; it is $\mathcal{L}$-superharmonic in $\mathbb{G}$ if and only if there exists $z \in \mathbb{G}$ such that $\Gamma_{\mu}(z)<\infty$, see [5, Theorem 9.3.2].

In this case, see [5, Theorem 9.3.5],

$$
\mathcal{L} \Gamma_{\mu}=-\mu \quad \text { in the sense of distributions }
$$

and

$$
\Gamma_{\mu} \text { is } \mathcal{L} \text {-harmonic in } \mathbb{G} \backslash \operatorname{supp} \mu \text {. }
$$

For our purposes, the following remark is crucial.

Remark 5.1. Let $\left(\Omega, \mu, x_{0}\right)$ be a $\Gamma$-triple (see Definition 2.2) and let $A \subseteq \Omega$ be a Borel set. Then the function

$$
\mathbb{G} \ni x \mapsto \Gamma_{\mu_{A}}(x):=\int_{A} \Gamma\left(x^{-1} \circ y\right) d \mu(y)
$$

is the $\Gamma$-potential of $\mu_{A}:=\mu\llcorner A$ and satisfies

$$
\Gamma_{\mu_{A}}(x) \leq \Gamma_{\mu}(x)=\Gamma\left(x^{-1} \circ x_{0}\right)<\infty \quad \forall x \in \Omega^{c} .
$$

Moreover, $\Gamma_{\mu_{A}}$ is $\mathcal{L}$-superharmonic in $\mathbb{G}$ and

$$
\Gamma_{\mu_{A}} \text { is } \mathcal{L} \text {-harmonic in } O
$$

for every open set $O \subseteq A^{c}$. Indeed $O \subseteq A^{c}$ implies $O \subseteq \bar{A}^{c} \subseteq\left(\operatorname{supp} \mu_{A}\right)^{c}$. 


\section{REFERENCES}

[1] D. Aharonov, M.M. Schiffer, L. Zalcman: Potato kugel, Israel J. Math. 40 (1981) 331-339.

[2] H. Aikawa: Integrability of superharmonic functions and subharmonic functions, Proc. Amer. Math. Soc. 120 (1994) 109-117.

[3] H. Alkawa: Densities with the mean value property for harmonic functions in a Lipschitz domain, Proc. Amer. Math. Soc. 125 (1997) 229-234.

[4] A. Bonfiglioli, E. Lanconelli: On left invariant Hörmander operators in $\mathbb{R}^{N}$. Applications to KolmogorovFokker-Planck equations, J. Math. Sci. 171 (2010), 22-33.

[5] A. Bonfiglioli, E. Lanconelli, F. Uguzzoni: Stratified Lie Groups and Potential Theory for their subLaplacians. Springer Monographs in Mathematics. Springer, Berlin, 2007.

[6] G. Cupini, E. Lanconelli: On an inverse problem in the Potential Theory, Atti Acc. Sci. Mat. Ren. Lincei to appear.

[7] B. Epstein: On the mean-value property of harmonic functions, Proc. Amer. Math. Soc. 13 (1962) 830.

[8] B. Epstein, M.M. Schiffer: On the mean-value property of harmonic functions, J. Analyse Math. 14 (1965) 109-111.

[9] M. Goldstein, W. Haussmann, L. Rogge: On the mean value property of harmonic functions and best harmonic $L^{1}$-approximation, Trans. Amer. Math. Soc. 305 (1988) 505-515.

[10] W. Hansen, I. Netuka: Volume densities with the mean value property for harmonic functions, Proc. Amer. Math. Soc. 123 (1995) 135-140.

[11] L. Hörmander: Hypoelliptic second order differential equations, Acta Mathematica 119 (1967), 147-171.

[12] Ü. KuRAN: On the mean-value property of harmonic functions, Bull. London Math. Soc. 4 (1972), 311-312.

[13] E. Lanconelli: "Potato kugel" for sub-Laplacians, Israel J. Math. 194 (2013) 277-283. 